\title{
Depressão, ansiedade, estresse e qualidade de vida de estudantes de universidades pública e privada
}

\author{
Depression, anxiety, stress and quality of life of \\ students from public and private universities
}

\section{Depresión, ansiedad, estrés y calidad de vida para estudiantes de universidades públicas y privadas}

\section{Michelle Firmino Guimarães ${ }^{1}$ (1) Marília Martins Vizzotto ${ }^{2}$ (1) Hilda Rosa Maria Capelão Avoglia 3 (1) Eliane Aparecida Faria Paiva4 ${ }^{4}$}

\author{
1'Autora para correspondência. Universidade Metodista de São Paulo (São Paulo). São Paulo, Brasil. psicologiainc@ufam.edu.br \\ 2-4Universidade Metodista de São Paulo (São Paulo). São Paulo, Brasil. mariliamartinsvizzotto@gmail.com, hildaavoglia@terra.com.br,
} eapaiva_psi@yahoo.com.br

\begin{abstract}
RESUMO | INTRODUÇÃo: Variáveis como depressão, ansiedade, estresse e qualidade de vida oferecem indicativos de saúde e doença de jovens, estudantes em fase de formação profissional, uma vez que representam quatro das dez principais causas de incapacidade no mundo. OBJETIVO GERAL: Avaliar e correlacionar a presença de sinais e sintomas de depressão, ansiedade, estresse e qualidade de vida de universitários de duas instituições, sendo uma pública e outra privada; OBJETIVOS: a) Caracterizar o perfil sociodemográfico; b) Avaliar sintomas de depressão, sinais de ansiedade e estresse e qualidade de vida geral; c) correlacionar as variáveis qualidade de vida, sinais e sintomas de ansiedade, depressão e estresse. MÉTODO: Pesquisa de cunho quantitativo. Para coleta de dados foram utilizados os seguintes instrumentos: questionário de dados socioeconômicos e culturais; WHOQOL Breve e EADS - Escala de Depressão, Ansiedade e Estresse Os dados foram avaliados estatisticamente com auxílio do Programa SPSS- versão 2.0 para Windows. RESULTADOS: Participaram da pesquisa 570 Universitários, 344 estudantes da Instituição Privada e 226 da Instituição Pública, dos gêneros feminino e masculino, entre 16 e 55 anos. Os resultados revelaram que não houve diferença significativa na qualidade de vida nos domínios Psicológico e Social de ambas as Instituições. Os domínios mais afetados, ou seja, aquele, em que os universitários encontram mais prejuízo é no que se referem ao domínio Físico e Meio Ambiente de ambas as Instituições. CONCLUSÃO: Assim sendo, observa-se que os estudantes obtiveram um grau leve de estresse, depressão e ansiedade em ambas as instituições. Ao correlacionar os domínios de qualidade de vida e as variáveis: ansiedade, depressão e estresse, observou-se que quanto maior a depressão, menor a qualidade de vida nos domínios psicológico e ambiental. A partir dos resultados, sugerem-se estudos mais aprofundados.
\end{abstract}

PALAVRAS-CHAVE: Qualidade de vida. Ansiedade. Depressão. Estresse

ABSTRACT | INTRODUCTION: Variables such as depression, anxiety, stress, and quality of life offer indicators of health and illness in young students undergoing professional training, as they represent four of the ten main causes of disability in the world. General. OBJECTIVE: Evaluate the correlation of the presence of signs and symptoms of depression, anxiety, stress, and quality of life of university students from two institutions, one public, and one private. OBJECTIVES: a) Characterize the socio-demographic profile; b) Assess symptoms of depression, signs of anxiety and stress, assess general quality of life; c) correlate the variables quality of life, signs, and symptoms of anxiety, depression, and stress. METHOD: Quantitative research. The following instruments were used for data collection: socioeconomic and cultural data questionnaire; Brief WHOQOL and EADS - Depression, Anxiety and Stress Scale Data were statistically evaluated using the SPSS Program - version 2.0 for Windows. RESULTS: 570 university students, 344 students from private and 226 from the public, male and female, aged between 16 and 55, participated in the research. The results revealed no significant difference in the quality of life in both institutions' Psychological and Social domains. The most affected domains, that is, the ones in which university students find the most damage, are related to both institutions' physical and Environmental domains. CONCLUSION: Therefore, it is observed that students obtained a mild degree of stress, depression, and anxiety in both institutions. When correlating the domains of quality of life and the variables: anxiety, depression, and stress, it was observed that the greater the depression, the lower the quality of life in the psychological and environmental domains. From the results, further studies are suggested.

KEYWORDS: Quality of life. Anxiety. Depression. Stress. 
RESUMEN | INTRODUCCIÓN: Variables como depresión, ansiedad, estrés y calidad de vida ofrecen indicadores de salud y enfermedad en jóvenes estudiantes en formación profesional, ya que representan cuatro de las diez principales causas de discapacidad en el mundo. OBJETIVO GENERAL: Evaluar y correlacionar la presencia de signos y síntomas de depresión, ansiedad, estrés y calidad de vida en estudiantes universitarios de dos instituciones, una pública y otra privada; OBJETIVOS: a) Caracterizar el perfil sociodemográfico; b) Evaluar síntomas de depresión, signos de ansiedad y estrés y calidad de vida en general; c) correlacionar las variables calidad de vida, signos y síntomas de ansiedad, depresión y estrés. MÉTODO: Investigación cuantitativa. Para la recolección de datos se utilizaron los siguientes instrumentos: cuestionario de datos socioeconómicos y culturales; Los datos breves de WHOQOL y EADS - Depresión, Ansiedad and Escala de Estrés se evaluaron estadísticamente utilizando el programa SPSS - versión 2.0 para Windows. RESULTADOS: La encuesta incluyó a 570 estudiantes universitarios, 344 estudiantes de la Institución Privada y 226 de la Institución Pública, hombres y mujeres, con edades comprendidas entre los 16 y 55 años. Los resultados revelaron que no hubo diferencia significativa en la calidad de vida en los dominios Psicológico y Social de ambas instituciones. Los dominios más afectados, es decir, aquél en el que los estudiantes universitarios encuentran más daño está relacionado con el dominio Físico y Ambiental de ambas Instituciones. CoNCLUSıón: Por lo tanto, se observa que los estudiantes obtuvieron un grado leve de estrés, depresión y ansiedad en ambas instituciones. Al correlacionar los dominios de calidad de vida y las variables: ansiedad, depresión y estrés, se observó que, a mayor depresión, menor calidad de vida en los dominios psicológico y ambiental. A partir de los resultados, se sugieren más estudios.

PALABRAS CLAVE: Calidad de vida. Ansiedad. Depresión. Estrés

\section{Introdução}

As preocupações com a saúde mental de estudantes tornaram-se ainda mais presentes quando nos deparamos com estudos que evidenciavam incidência de distúrbios psicológicos e psiquiátricos na população geral, sendo que esses representam quatro das dez principais causas de incapacitação mundial.

A depressão é a principal causa de incapacidade no mundo, sendo um transtorno mental muito frequente que, segundo a Organização Mundial da Saúde (OMS) (2020), atinge mais de 264 milhões de pessoas no mundo. Morrem, a cada ano, aproximadamente 800 mil pessoas por suicídio atribuído à depressão, sendo essa a segunda maior causa de morte de pessoas com idades entre 15 e 29 anos.

Os problemas de saúde mental já são considerados as doenças do século 21, com destaque para a depressão, que poderá ser a doença mais comum do mundo até 2030, segundo a OMS (Sociedade Brasileira de Clínica Médica, 2009).

No Brasil, a Rede de Cuidados em Saúde Mental, Crack, Álcool e outras Drogas foi pactuada em julho de 2011, como parte das discussões de implantação do Decreto n 7508, de 28 de junho de 2011, e prevê, a partir da Política Nacional de Saúde Mental, os Centros de Atenção Psicossocial (CAPs), os Serviços Residenciais Terapêuticos, os Centros de Convivência e Cultura, as Unidades de Acolhimento e os leitos de atenção integral em Hospitais Gerais. (Ministério da Sáude, 2011).

Segundo o Ministério da Saúde, no Brasil a incidência de doenças mentais na população é de 23 milhões de brasileiros (12\% da população necessita de algum atendimento em saúde mental); pelo menos 5 milhões de brasileiros (3\% da população) sofrem com transtornos mentais graves e persistentes.

No estudo sobre o resultado do inquérito Megacity Mental Health Surve em relação a prevalência de transtornos mentais distribuídos em idade e sexo da região metropolitana de São Paulo mostrou que a região metropolitana de São Paulo possui a maior incidência de perturbações mentais no mundo. $O$ estudo revelou que $44,8 \%$ dos paulistanos, e moradores da região metropolitana de SP, sofrem de algum tipo de perturbação mental. De acordo com os pesquisadores responsáveis pelo estudo, a alta incidência de perturbações mentais é causada pela alta urbanização associada com privações sociais. Segundo eles, os grupos mais vulneráveis são homens migrantes e mulheres que residem em regiões de alta vulnerabilidade social (Viana \& Andrade., 2012).

Assim, variáveis como ansiedade, depressão e estresse, bem como o nível de qualidade de vida estão implicados nesse conceito amplo e de difícil conceituação e de descrição. De modo que, os estudos que dão indicativos de estresse, de ansiedade e depressão, estarão todos buscando relações com essa saúde mental. 
Para que se tenha uma melhor compreensão do tema é necessário trazer algumas definições. Começaremos pela compreensão de Qualidade De Vida (QV).

No estudo realizado por Lopes Neto et al. (2009) sobre a Atenção à Saúde Mental no Amazonas, Brasil: um olhar sobre os Centros de Atenção Psicossocial, as informações desvelam que a demanda de pessoas com transtornos mentais é significativa e existem apenas três CAPS para uma população de mais de três milhões habitantes, distribuídas $60 \%$ na capital e 40\% nos demais 61 municípios, requerendo um olhar dos gestores em nível municipal, estadual e federal, haja vista que os achados revelam uma fragilidade da organização da rede de saúde mental pela escassez de recursos físicos, materiais e humanos. Na cidade do Amazonas em que está sendo realizada a pesquisa ainda não existe o CAPS.

Além disso, o Estado da realização da pesquisa e o Brasil como um todo carecem de informações dessa natureza e que sejam baseadas em dados de pesquisa científica. Como relata Baldassin (2010), estudos brasileiros sobre sintomas de ansiedade, depressão, estresse e burnout ainda são poucos, basicamente transversais e não multicêntricos.

\section{Qualidade de vida}

O conceito de Qualidade de Vida sofreu evolução ao longo do século $X X$, tendo em vista as divergências, especulações e os estudos acerca do significado e sentido do termo. Hoje, conforme Almeida et al., (2012) a qualidade de vida constitui um importante campo para o diálogo entre as diferentes disciplinas e escolas de pensamento, no sentido da busca de avanços reais para as pessoas das mais diferentes culturas. Entretanto, é interessante também comentar que o senso comum se apropriou desse objeto de forma a resumir melhorias ou um alto padrão de bem-estar na vida das pessoas, sejam elas de ordem econômica, social ou emocional.

Todavia, a área de conhecimento em Qualidade de Vida encontra-se em uma fase de construção, pois a identificam ora em relação à saúde, ora à moradia, ao lazer, aos hábitos de atividades físicas e alimentação; mas, o fato é que essa forma de saber afirma que todos esses fatores levam a uma percepção positiva de bem-estar (Marques, 2007).

Com o final da Segunda Grande Guerra, a Organização das Nações Unidas (ONU), preocupada com as condições de vida das pessoas dos países membros, fez uma recomendação para que fossem estudadas as variáveis que interfeririam nessas condições de vida. Assim, surgem na década de 1950 investigadores, clínicos, epidemiologistas, cientistas sociais e estatísticos, conduzindo pesquisas de medidas de atributos humanos, denominados de "boa vida" (Oliveira et al., 2011).

Segundo esses mesmos autores o conceito de "boa vida" foi usado com significado de conquista de bens materiais (possuir casa própria, carro, aparelhos eletrônicos e outros) sendo, posteriormente, ampliada para avaliar o quanto uma sociedade havia se desenvolvido economicamente, não importando se tal riqueza estava bem distribuída, um problema ainda atual na maioria dos países, onde há uma grande concentração de riquezas nas mãos de poucos.

Do ponto de vista histórico, tal como citado por Fleck et al., (1999) a expressão "qualidade de vida" foi empregada pelo presidente dos Estados Unidos, Lyndon Johnson, em 1964, ao declarar que os objetivos não podiam mensurar pelos saldos das casas bancárias. Eles só poderiam ser medidos através da qualidade de vida proporcionada às pessoas. $\mathrm{O}$ interesse em conceitos como "padrão de vida" e "qualidade de vida" foi inicialmente compartilhado por cientistas sociais, filósofos e políticos. $\mathrm{O}$ crescente desenvolvimento tecnológico da Medicina e ciências afins trouxe como uma consequência negativa a sua progressiva desumanização. Assim, a preocupação com o conceito de "qualidade de vida" refere-se a um movimento dentro das ciências humanas e biológicas, exatas e engenharia no sentido de valorizar parâmetros mais amplos do que o simples controle de sintomas, e diminuição da mortalidade ou o aumento da expectativa de vida.

Para chegar a um conceito, a Organização Mundial de Saúde criou um grupo destinado a conceituar "qualidade de vida". Assim, o Grupo de Qualidade de Vida da divisão de Saúde Mental da OMS definiu a "qualidade de vida" como "a percepção do indivíduo de 
sua posição na vida no contexto da cultura e sistema de valores nos quais ele vive e em relação aos seus objetivos, expectativas, padrões e preocupações" (OMS, 1998, p. 04). Após tal definição é que os autores dos mais diversos campos de atuação deram seguimento aos estudos sobre o tema. De modo que o conceito se ampliou para significar além do crescimento econômico, o desenvolvimento social (saúde, educação, moradia, transporte, lazer, trabalho, crescimento individual) e, assim, pôde descrever com maior proximidade e veracidade as condições de vida da população.

\section{Ansiedade}

Segundo a Classificação Estatística Internacional de Doenças e Problemas relacionados à Saúde- $10^{\text {a }}$ Revisão (CID 10, 2008), no transtorno de ansiedade generalizada o aspecto essencial é ansiedade, a qual é generalizada e persistente, mas não restrita ou mesmo fortemente. O paciente deve ter sintomas primários de ansiedade na maioria dos dias por pelo menos várias semanas e usualmente por vários meses. Esses sintomas devem usualmente envolver: apreensão (preocupação sobre desgraças futuras e sentir-se no limite, dificuldade de concentração); tensão motora (movimentação inquieta, cefaleias tensionais, tremores, incapacidade de relaxar) e hiperatividade autonômica (sensação de cabeça leve, sudorese, taquicardia ou taquipneia, desconforto epigástrico, tonturas e boca seca).

Para Parreira (2008), ansiedade é o conjunto de sinais e sintomas que abrangem do campo físico e emocional, do mental ao existencial. No nível físico, existe uma relação entre ansiedade e reação fisiológica chamada de estresse. Diante de um agente agressor externo, o organismo reage colocando-se em posição de defesa, pronto para lutar ou fugir, dependendo do contexto. Os batimentos cardíacos aumentam, enquanto o sangue sai da superfície para irrigar melhor os músculos. A respiração fica mais rápida e superficial e as pupilas se contraem.

Sentir ansiedade é no mínimo desconfortável, consistindo-se em uma mistura de sentimentos e sensações que perturbam nosso cotidiano, roubando boa parte da energia que seria empregada de outras formas, desde o trabalho até a diversão. Mas apesar de não estar associada ao prazer, a ansiedade não é algo ruim, um mau sinal. O problema é o desequilíbrio desse mecanismo, tanto no excesso quanto na falta. Em demasia a ansiedade pode acontecer um estresse improdutivo. Por outro lado, sua ausência é equivalente a um organismo debilitado que não consegue se defender (Parreira, 2008).

\section{Depressão}

No Relatório sobre Saúde no Mundo da OMS (2001) em sua abordagem sobre os transtornos depressivos, passa a relatar que a Depressão se caracteriza por tristeza, perda de interesse em atividades e diminuição da energia. Com relação aos sintomas presentes estão a perda de confiança e autoestima, o sentimento injustificado de culpa, ideias de morte e suicídio, diminuição da concentração e perturbações do sono e do apetite. Podem estar presentes também diversos sintomas somáticos.

A depressão foi descrita e definida pela American Psychiatric Association (Diagnostic and Statistical Manual of Mental Disorders, 2002) como transtorno de humor que envolve um grupo heterogêneo de sintomas: humor deprimido, interesse ou prazer acentuadamente diminuído, perda ou ganho significativo de peso, insônia ou hipersonia, agitação ou retardo psicomotor, fadiga ou perda de energia, sentimento de inutilidade ou culpa excessiva ou inadequada, capacidade diminuída de pensar e pensamentos de mortes recorrentes.

Por que estamos mais deprimidos do que nunca $O$ psicólogo Jean Twenge descobriu que os aumentos da depressão nos últimos 50 anos correspondem a um aumento no individualismo e a uma perda de conectividade social. (Leahy, 2015).

No século XIX quase ninguém vivia sozinho, no entanto hoje, cerca de $26 \%$ dos lares são formados por uma pessoa. A solidão e problemas de relacionamento contribuem para a Depressão. A Depressão é uma epidemia mundial que priva as vidas de significado e pode até matar (Leahy, 2015). 


\section{O estresse na vida acadêmica}

A vida contemporânea está cada vez mais estressante. Assaltos, mudanças de valores, tecnologia avançada, aumento de desestruturação familiar, excesso de competição e muitos outros fatores externos e internos contribuem para que o ser humano viva constantemente em um alto nível de estresse (Lipp, 2007). E o contexto acadêmico estudado, no caso os estudantes universitários vivenciam toda essa turbulência como relata (Calais et al., 2007) no parágrafo seguinte.

O ingresso na universidade pode colocar os alunos em contato com estressores específicos, tais como medo, ansiedade e inseguranças geradas pelas possíveis dúvidas e desilusões com a carreira escolhida, aquisição de novas e maiores responsabilidades, problemas na moradia, distanciamento da família, dificuldade no estabelecimento de relacionamento afetivo (Calais et al., 2007). Em alguns momentos e situações da vida acadêmica podem ser caracterizados por um ambiente estressante.

Deste modo, presente estudo investigou os seguintes objetivos:

a) Avaliar a presença de sinais e sintomas de depressão, ansiedade, estresse e qualidade de vida de universitários de duas instituições sendo uma pública e outra privada;

b) Caracterizar o perfil sociodemográfico desses universitários de ambas as instituições;

c) Correlacionar as variáveis qualidade de vida, sinais e sintomas de ansiedade, depressão e estresse entre os estudantes de ambas as instituições.

\section{Método}

Participantes: A amostra inicial era estimada de 400 estudantes, sendo 200 de cada instituição e no final participaram da pesquisa 570 universitários, sendo 344 da Instituição Privada) e 226 da Instituição Pública), estudantes de diferentes cursos universitários. Foram amostras casuais e por conveniência, conforme preconizaram Rea e Parker (2000), estudantes dos cursos de Antropologia, Pedagogia, Letras, Ciências Agrárias, Biologia/Química e Administração,
Psicologia, Biomedicina, Medicina Veterinária e Gestão em Recursos Humanos.

Foram incluídos na amostra universitários devidamente matriculados e frequentando a Universidade de diversos cursos, de ambos os sexos/idades, que aceitaram e autorizaram sua participação por meio da concordância com o TCLE.

Foram excluídos da amostra: alunos não matriculados e frequentando os cursos da Universidade ou que se recusaram a assinar o TCLE.

Local: A coleta de dados foi realizada em salas de aula, em ambas as Instituições: pública e privada; em horários de aula e a partir da permissão do docente responsável e após autorização das instituições para esse levantamento.

Pesquisa de cunho quantitativo. O Método para coleta de dados foram os seguintes instrumentos: Questionários e Escala. A sequência de aplicação dos instrumentos foi a seguinte: 1) Questionário de Dados Sociodemográficos e Cultural, 2) Escala de Ansiedade, Depressão e Estresse e 3) Questionário Whoqol Breve.

Procedimentos: Os dados foram coletados na Instituição Pública em dezembro de 2012 nos períodos matutino e vespertino. Na Universidade Privada, nos meses de março, abril, outubro e novembro de 2013, nos turnos diurno e noturno. Os instrumentos, autoaplicáveis, foram feitos em salas de aula após aprovação do Comitê de Ética de Pesquisa de ambas as Instituições), mediante autorização das Instituições e permissão do docente responsável. (Parecer datado em 22/11/2012).

A coleta de dados foi realizada após aprovação do Comitê de Ética em Pesquisa de ambas as Universidades. O estudo seguiu as determinações éticas sobre pesquisas com seres humanos de acordo com a resolução n¹96/96 do Conselho Nacional de Saúde.

Os instrumentos foram aplicados pela autora da presente pesquisa que é psicóloga, no espaço da sala de aula da Universidade de forma presencial e coletiva após os participantes assinarem e concordarem com o Termo de Consentimento Livre e Esclarecido (TCLE). 
O projeto de Pesquisa passou pelo Comitê de Ética da Universidade (Plataforma Brasil) e foi aprovado para realização da pesquisa nas Instituições de Ensino, devido envolver seres humanos coletivamente, de forma direta ou indireta. Há uma preocupação pela integridade e bem-estar dos sujeitos da pesquisa. O projeto obedeceu às normas da Resolução n¹96/96 que aprova as diretrizes e normas regulamentadoras de pesquisas envolvendo seres humanos. (Parecer: 160099, CAAE: 10644412.4.1001.5508 datado em 22/11/2012).

Foram orientados em relação aos riscos que a pesquisa poderia trazer algum desconforto ou risco mínimo para a saúde emocional. Caso isso ocorresse, a pesquisadora estaria a disposição para a atenção psicológica devida e gratuita, ou seja, atendimento e encaminhamento caso necessário uma vez que ela é psicóloga, devidamente credenciada no Conselho Regional de Psicologia sob o número CRP 20/02644.

Benefícios: O participante da pesquisa poderia receber uma devolutiva quando terminasse a pesquisa e caso manifeste interesse em conhecer seus resultados.

Foi lhe assegurado o anonimato e que poderiam desistir de participar da pesquisa há qualquer momento.

\section{Resultados e discussão}

\section{1) Características Socioculturais e demográficas dos universitários}

Na Tabela abaixo observam-se que a idade média dos estudantes da Universidade Pública é de 24,23 anos ( $D P=6,67)$ e dos alunos da Universidade Privada é de 23,37 anos ( $D P=6,18)$, sendo o máximo 55 anos e mínimo 16 anos. A presença significante de solteiros está de acordo com o esperado e com a idade média foi 24 anos, já que o estado civil costuma está associado à idade. Quanto à renda familiar dos participantes, a renda média dos universitários da Universidade Pública foi $R \$ 369,00$ e da Universidade Privada foi $R \$ 1388,70$. A renda familiar média foi de padrão modesto entre os estudantes de ambas as universidades. Rossetti Junior e Schimiguel (2011) que estudaram perfil dos estudantes dessa região sudeste verifica-se muita proximidade, pois os dados dos autores apontaram para uma renda familiar declarada inferior a $\mathrm{R} \$ 2.000,00$, indicando uma renda modesta para alunos de instituição particular cuja mensalidade é de aproximadamente $R \$ 420,00$. Outro aspecto importante de ser discutido é o fato de que a PNAD (2011) ter observado em seus levantamentos que as universidades públicas absorvem as camadas mais ricas da população; todavia, em relação à especificidade dessa região amazônica em comparação com sudeste, essa não foi uma regra. Assim, entendemos que há sempre a necessidade de não generalização quando tratamos de especificidades regionais e culturais num país tão grande quanto o nosso.

Em relação à escolaridade da mãe e do pai "sem estudos" ou "ensino primário" houve diferenças significativas entre as instituições, apontando para o fato de que os jovens da Universidade Pública são oriundos de famílias com menos escolaridade. 
Tabela 1. Distribuição por sexo, Idade, Estado civil, opção de curso, mudança de residência,escolaridade dos pais e Renda dos universitários da amostra ou Distribuição dos dados sociodemográficos e culturais dos universitários da amostra

\begin{tabular}{llll}
\hline VARIÁVEIS & NÍVEIS & UFAM & UMESP \\
\hline Sexo & Feminino & $145-25,4 \%$ & $50-8,8 \%$ \\
& Masculino & $81.14,2 \%$ & $284-51,6 \%$ \\
\hline Idade & Mínimo & 55 & 53 \\
& Máximo & 16 & 17 \\
& & & \\
\hline Estado Civil & Solteiro & $188-33 \%$ & $295-52 \%$ \\
& Casados & $22-3,9 \%$ & $42-7,4 \%$ \\
\hline Opção pelo curso & $1^{\circ}$ opção & $138-25,2 \%$ & $225-41 \%$ \\
& $2^{\circ}$ opção & $61-11 \%$ & $79-14,4 \%$ \\
\hline Mudou de residência & Não & $156-27,5 \%$ & $317-55,8 \%$ \\
& Sim & $66-11,6 \%$ & $25-4,4 \%$ \\
\hline Escolaridade dos pais & Primário & $50-8,9 \%$ & $44-7,8 \%$ \\
& Fundamental & $59-10,5 \%$ & $69-12,3 \%$ \\
& Médio & $60-10,7 \%$ & $102-18,2 \%$ \\
& Superior & $23-4,1 \%$ & $115-20,5 \%$ \\
\hline Renda & Média & $\mathrm{R} \$ 369,00$ & $\mathrm{R} \$ 1389,00$ \\
\hline
\end{tabular}

\section{2) Qualidade de vida dos universitários}

Tabela 2. Qualidade de vida de ambos os grupos de universitários

\begin{tabular}{lll}
\hline Domínios de QV & UFAM (Média) & UMESP (Média) \\
\hline Físico & 54.70 & 51.00 \\
\hline Psicológico & 70.80 & 70.00 \\
\hline Social & 70.80 & 69.30 \\
\hline Ambiental & 58.60 & 59.00 \\
\hline
\end{tabular}

Os melhores níveis de QV encontraram-se nos domínios Psicológico e Social em ambas as instituições. Os domínios mais afetados foram os domínios físico e meio ambiente entre estudantes de ambas as Instituições estudadas. Os estudantes da UFAM e UMESP estão satisfeitos com as suas vidas no âmbito afetivo, emocional, relações sociais e atividade sexual.

Essa percepção mais prejudicada no domínio físico pode estar relacionada, tal como sugeriram Petrini et al. (2011) com o excesso de realização de tarefas cotidianas e corriqueiras desses estudantes. Hoje em dia são muitos os jovens que trabalham e estudam, assumindo assim uma dupla jornada.

Por outro lado, esses resultados são contrários aos achados de Barreto et al. (2009) em estudantes africanos residentes em João Pessoa. Também em relação ao estudo de Souza et al. (2012) que compararam a qualidade de vida de estudantes ingressantes e concluintes de educação física. Estes dois estudos indicaram um nível alto de Qualidade de Vida no domínio físico entre estudantes. 
Tabela 3. Domínios da qualidade de vida e sexo da amostra estudada ( $n=570)$

\begin{tabular}{lll}
\hline Domínios & Sexo & Média \\
\hline Físico & Masculino & 53.70 \\
& Feminino & 52.00 \\
\hline Psicológico & Masculino & 70.17 \\
& & 67.36 \\
& Feminino & \\
\hline Social & Masculino & 72.13 \\
& Feminino & 69.21 \\
\hline Ambiental & Masculino & 55.28 \\
& Feminino & 56.09 \\
\hline
\end{tabular}

Os dados revelaram que em relação ao sexo, as mulheres têm menor qualidade de vida do que os homens. Em especial, verifica-se que os homens tiveram melhores resultados nos domínios físico, psicológico e social.

Esses resultados são semelhantes aos achados por Andrade et al., (2011) sobre estudantes do curso de Psicologia de Campo Grande, Mato Grosso do Sul em que os homens tiveram QV melhor do que as mulheres.

Entretanto, dados diferentes foram encontrados por Meyer et al., (2012) que verificaram não haver diferença significativa nos escores de qualidade de vida e estresse ocupacional entre os sexos de uma amostra de estudantes de medicina, universitários de instituições públicas e privadas de Santa Catarina. De forma mais detalhada, os autores encontraram que as mulheres tinham melhor qualidade de vida em relação às questões sociais e ambientais e concluíam que o sexo feminino é mais solidário, e as mulheres são mais sociáveis e possuem essas características positivas relacionadas à satisfação com a vida e às relações sociais.

\section{4) Ansiedade, depressão e estresse do sexo da amostra}

Tabela 4. Ansiedade, depressão e estresse do sexo da amostra

\begin{tabular}{lllllll}
\hline \multicolumn{1}{c}{ Sexo } & \multicolumn{3}{c}{ Masculino } & \multicolumn{3}{c}{ Feminino } \\
Variáveis & Média & Mínimo & Máximo & Média & Mínimo & Máximo \\
. Ansiedade & 6,61 & 00 & 26 & 8,41 & 00 & 42 \\
. Depressão & 8,16 & 00 & 36 & 9,69 & 00 & 42 \\
. Estresse & 11,61 & 00 & 34 & 15,00 & 00 & 42 \\
\hline
\end{tabular}

Pode-se observar que a maior média apresentada se refere ao fator estresse, do sexo feminino, sendo representado pelo resultado de 15,00 e a média menor foi no fator ansiedade masculina (6,61). Assim, avalia-se que as mulheres estão mais estressadas; e com relação às demais variáveis, também as mulheres se apresentam mais ansiosas e depressivas do que os homens.

Assim, observa-se que em todas as variáveis as mulheres tiveram mais altos escores. Este aspecto vem a coincidir com resultados de Apóstolo et al. (2011) que analisaram a prevalência pontual de depressão, ansiedade e estresse em usuários de um Centro de Saúde de Portugal e identificaram níveis muito graves de estresse e níveis graves de ansiedade de depressão, sendo as mulheres aquelas com níveis elevados de estresse e médios de depressão e ansiedade. Também o estudo de Gama et al. (2008) que utilizaram Inventário de Ansiedade Traço-Estado em uma amostra de estudantes universitários e encontraram maiores níveis de ansiedade entre mulheres, solteiras e até 30 anos. 
Entretanto, o Relatório sobre Saúde Mundial feito pela OMS (2001) revela que a maioria dos estudos chegou à conclusão de que a prevalência geral de transtornos mentais é aproximadamente a mesma no sexo masculino e no feminino. Algumas diferenças existentes são explicadas pela distribuição diferencial dos transtornos; mas, os transtornos mentais graves são igualmente comuns, com exceção da depressão, que é mais comum no sexo feminino, e dos transtornos devidos ao uso de substâncias, que ocorrem mais frequentemente no sexo masculino.

\section{5) Correlação entre depressão. Estresse e ansiedade de ambos os grupos de universitários}

Tabela 5. Correlação entre depressão, estresse e ansiedade de ambos os grupos de Universitários (r Person)

\begin{tabular}{lllll}
\hline Variáveis & Instituição & Depressão & Estresse & Ansiedade \\
\hline Depressão & UMESP & - & $\mathbf{0 , 7 3}$ & $\mathbf{0 , 7 1}$ \\
Estresse & UMESP & $\mathbf{0 . 7 3}$ & - & $\mathbf{0 , 7 6}$ \\
Ansiedade & UMESP & $\mathbf{0 , 7 1}$ & $\mathbf{0 , 7 6}$ & - \\
\hline Depressão & UFAM & - & $\mathbf{0 , 6 6}$ & $\mathbf{0 , 7 0}$ \\
Estresse & UFAM & $\mathbf{0 , 6 6}$ & - & $\mathbf{0 , 7 3}$ \\
Ansiedade & UFAM & $\mathbf{0 , 7 0}$ & $\mathbf{0 , 7 3}$ & \\
\hline
\end{tabular}

Os resultados revelaram índices de correlação positiva e significativa entre as três variáveis de estresse/ansiedade, ansiedade/depressão e depressão/estresse/ansiedade dos universitários da UMESP e da UFAM. Os dados indicam que quanto maior a depressão, maior o estresse, maior a ansiedade e vice-versa.

No estudo em questão houve correlação significativa entre ansiedade e depressão, embora não tenha sido a mais forte, pois segundo Ribeiro et al. (2004), a ansiedade costuma estar estreitamente associada aos sintomas de depressão e, até finais do século XIX as perturbações de ansiedade não se separavam de outras perturbações do humor.

6) Correlação geral entre os domínios e ansiedade, depressão e estresse -amostra total (n=570)

Tabela 6. Correlação geral entre os domínios e ansiedade, depressão e estresse da amostra total (n=570)

\begin{tabular}{llllll}
\hline Variáveis & Geral & Físico & Psicológico & Social & Ambiental \\
\hline Ansiedade & $-0,20$ & $-0,10^{* *}$ & $-0,19^{\star *}$ & $-0,20^{*}$ & $-0,30^{* *}$ \\
\hline Depressão & $-0,20^{* *}$ & $-0,17^{* *}$ & $-0,32^{* *}$ & $-0,23^{* *}$ & $-0,25^{* *}$ \\
\hline Estresse & $-0,19^{\star *}$ & $-0,16^{*}$ & $-0,23^{\star *}$ & $-0,19^{*}$ & $-0,24^{\star *}$ \\
\hline
\end{tabular}

Os dados revelaram que a correlação (no total da amostra) entre qualidade de vida (overall) e ansiedade, depressão e estresse foram estatisticamente negativos. Houve correlação negativa alta entre depressão e domínio psicológico $\left(r=-0,32^{* *}\right)$, isso indica que quanto maior a depressão menor a qualidade de vida - no domínio psicológico. Verificou-se correlação, porém baixa entre ansiedade e domínio físico $\left(r=-0,10^{* *}\right)$. Houve correlação alta entre ansiedade e o domínio ambiental $(r=-0,30 * *)$, indicando que quanto maior a ansiedade menos recursos para lidar com as questões de: segurança física e proteção, ambiente no lar, recursos financeiros, cuidados de saúde. 
O domínio psicológico tal como explicaram Fleck et al., (2003) está relacionado às variáveis psicológicas como: autoestima, sentimentos, pensamentos, aprendizagem, memória e concentração, imagem corporal e aparência, espiritualidade, religiosidade, crenças pessoais. Portanto, há indicativos de que tais variáveis (depressão e domínio psicológico de QV) estão relacionadas, ainda que não atinjam percentuais preocupantes, atenta-se para o fato de que as características da depressão, como fadiga, sentimento de culpa, baixa autoestima, provocam a exacerbação dos sintomas e prejudicam as estratégias de enfrentamento do sujeito diante da vida e acontecimentos diários. Além disso, o transtorno depressivo ao longo da vida (Moreno et al., 2012) incapacita parcial ou totalmente as relações pessoais, no trabalho e no lazer. O mesmo se pode referir à QV em seu domínio psicológico, o qual quando prejudicado, dá indicativos de baixa autoestima, sentimentos e pensamentos pessimistas, dificuldades de memória e concentração, além de crenças pessoais de desânimo e pessimismo.

Deste modo, também é importante nessa questão sobre as relações entre sinais de depressão e qualidade de vida baixa (domínio psicológico), pois isso indica a eficácia dos instrumentos de avaliação (EADS). E, sobre essas relações entre o domínio psicológico e os indicativos depressivos, Souza e Cruz (2008) observaram em estudo com estudantes universitários portugueses, as narrativas dos sujeitos deprimidos evidenciavam referências a acontecimentos de vida negativos, e os mais frequentes foram a vivência de doença, o fim de uma relação amorosa, a morte de uma figura afetivamente significativa e as emoções negativas associadas.

\section{7) Correlação geral entre os domínios e ansiedade, depressão e estresse -amostra de estudantes da Instituição PÚBLICA, n=226}

Tabela 7. Correlação entre os domínios de QV e ansiedade, depressão e estresse (amostra de estudantes da Instituição Pública, n=226)

\begin{tabular}{llllll}
\hline Variáveis & Geral & Físico & Psicológico & Social & Ambiental \\
\hline Ansiedade & $-0,23$ & $-0,11^{\star \star}$ & $-0,14^{\star \star}$ & $-0,13^{\star}$ & $-0,34^{\star \star}$ \\
\hline Depressão & $-0,20^{\star \star}$ & $-0,19^{\star \star}$ & $-0,27^{\star \star}$ & $-0,19^{\star \star}$ & $-0,28^{\star \star}$ \\
\hline Estresse & $-0,20^{\star \star}$ & $-0,14^{\star}$ & $-0,19^{\star \star}$ & $-0,15^{\star}$ & $-0,30^{\star \star}$ \\
\hline
\end{tabular}

Observa-se na tabela uma correlação negativa entre os domínios de qualidade de vida e ansiedade, depressão e estresse dos Universitários da Instituição UFAM. Os dados revelaram uma maior correlação entre ansiedade e domínio ambiental (UFAM) ( $r=-0,34 * *)$, e posteriormente depressão e o domínio ambiental $(r=-0,28 * *)$, depressão e domínio psicológico $\left(r=-0,27^{* *}\right)$ e estresse-domínio ambiental $(r=-0,30 * *)$. Esses dados nos levam a apontar que quanto maior a depressão, ansiedade e estresse menores serão os domínios de qualidade de vida (psicológico, social e ambiental). Ou seja, quanto mais deprimidos, ansiosos e estressados estiverem esses jovens participantes, menor será sua qualidade de vida em todos os seus domínios e vice-versa.

A região onde habitavam o grupo de estudantes da Universidade Pública possui problemas específicos relacionados aos transportes, pois em primeiro lugar há distância considerável da capital, e há dificuldades em vias de acesso para outras regiões próximas. Com isso, o fato de o acesso à região ser limitado e dificultado, pode ser visto pelos jovens como meio ambiente também desfavorável. 
8) Correlação geral entre os domínios e ansiedade, depressão e estresse -amostra de estudantes da Instituição UMESP, $\mathrm{n}=344$

Tabela 8. Correlação entre os domínios de QV e ansiedade, depressão e estresse (amostra de estudantes da Instituição Privada, n=344)

\begin{tabular}{llllll}
\hline Variáveis & Geral & Físico & Psicológico & Social & Ambiental \\
\hline Ansiedade & $-0,17$ & $-0,11^{* *}$ & $-0,25^{* *}$ & $-0,25^{*}$ & $-0,27^{* *}$ \\
\hline Depressão & $-0,20 * *$ & $-0,17^{* *}$ & $-0,38^{* *}$ & $-0,27^{* *}$ & $-0,23^{* *}$ \\
\hline Estresse & $-0,20 * *$ & $-0,14^{*}$ & $-0,23^{* *}$ & $-0,22^{*}$ & $-0,26^{* *}$ \\
\hline
\end{tabular}

Verificamos que ao correlacionar os domínios (físico, psicológico, social e ambiental) com ansiedade, depressão e estresse da Instituição UMESP, obtivemos a maior correlação entre depressão e os domínios psicológico $\left(r=-0,38^{* *}\right)$ e social $\left(r=-0,27^{* *}\right)$. Isso representa que quanto maior o valor da depressão, menor será a qualidade de vida no domínio psicológico e social, ou seja, maior a depressão, menor a autoestima, suporte social e atividade sexual.

Essa correlação é de importante observação, tendo em vista que a própria OMS (2001) em relatório sobre Saúde no Mundo, descreve que doenças "mentais" e "físicas" são influenciadas por uma combinação de fatores biológicos, psicológicos e sociais; ademais, reconhece que os pensamentos, os sentimentos e o comportamento exercem significativo impacto na saúde física. Da mesma forma, reconhece-se que a saúde física exerce considerável influência sobre a saúde e o bem-estar mental. Fatores que estão todos relacionados entre si e que, em nossa hipótese, irá depender do tipo de amostra estudada e, portanto, da capacidade das pessoas e grupos lidarem com situações adversas em suas vidas.

Os estudantes da universidade privada, também perceberam seu meio ambiente como desfavorável e apresentaram relação com ansiedade. Entretanto, a região dispõe de todos os possíveis recursos modernos ou pós-modernos. Além disso, a mais rica faz divisa com a cidade do país em desenvolvimento tecnológico e recursos financeiros. Têm à disposição imensa variedade de atrações culturais e de lazer. Entretanto, a região apresenta sérios problemas com transporte e locomoção, dificuldades com a preservação da cultura, da história local/regional e problemas com habitação, dado à grande migração de pessoas que vieram em busca de trabalho, formando-se extensas áreas de habitação pobres "favelas".

\section{Considerações finais}

O presente estudo buscou avaliar e correlacionar a presença de sinais e sintomas de ansiedade, depressão, estresse e qualidade de vida de universitários de duas instituições (pública e privada), bem como caracterizar o perfil sociodemográfico dessa amostra de universitários de diferentes regiões do país (norte e sudeste).

Assim, a amostra de 570 universitários com média etária de 24 anos, maioria mulheres, solteiras, que viviam com suas famílias. Esses estudantes com renda familiar modesta, oriundos, em maioria, de famílias cujos pais tinham pouco estudo e que compunham uma camada da população em ascensão social, apresentaram qualidade de vida geral média para boa, e sinais leves de ansiedade, depressão e estresse. Com relação ao sexo, os domínios de qualidade de vida e os sinais de ansiedade, depressão e estresse observou-se que as mulheres estavam mais ansiosas, depressivas e estressadas e mais baixo nível de qualidade de vida do que os homens. 
Quando avaliados os domínios de qualidade de vida observou-se que os mais afetados, ou seja, aqueles que os universitários percebiam como mais prejudicados foram os domínios físico e ambiental de ambas as Instituições estudadas. Quando comparados os domínios de qualidade de vida com as variáveis - ansiedade, depressão e estresse entre os dois grupos de estudantes, pode-se verificar: que em ambas o domínio psicológico teve forte correlação com depressão, indicando que em ambos os grupos os estudantes tendiam a estar pouco satisfeitos consigo mesmos, havia presença de sentimentos negativos, humor deprimido, tendiam a ter uma visão negativa de si, do mundo e se sentirem insatisfeitos com vários aspectos de sua vida. Também se verificou que as variáveis - ansiedade, depressão, estresse estiveram fortemente relacionados com o domínio meio ambiente, principalmente entre os estudantes amazonenses; enquanto entre os estudantes paulistas o domínio meio ambiente teve forte relação com ansiedade.

Essa relação de estados psicológicos com o meio ambiente mereceu atenção, tendo em vista que mesmo estando em diferentes regiões, os jovens perceberam-se insatisfeitos com meio ambiente. Levantou-se com isso a hipótese de que, mesmo que por razões ambientais e regionais distintas, ambas as regiões impunham insatisfações. Os jovens estudantes da Universidade Pública poderiam perceber problemas específicos relacionados ao transporte, lazer, distância considerável da capital, falta de recursos, inclusive de vias de acesso para outras regiões; além disso, a conservação e manutenção ambiental das populações ribeirinhas são pouco vistas ou valorizadas e dão lugar à valorização das culturas hegemônicas; o que pode proporcionar ao jovem um desejo pelos recursos do mundo cosmopolita (grandes centros de compras, teatros, bares, cinemas), e que pode então visualizar seu meio ambiente como desfavorável. Além disso, esses jovens, estudantes, enfrentam na realidade, grandes dificuldades com o transporte para chegarem até a universidade, todos os dias, pela precariedade da região e pela real dificuldade geográfica de acesso.

Foi possível perceber uma fragilidade presente nesses jovens estudantes e, esse quadro necessita de ganhar maior relevância no contexto acadêmico-científico e clínico, não só em razão do crescimento da população acadêmica em face à maior facilidade de acesso à universidade, mas também por seu significado em termos de saúde pública.

A partir destes resultados, sugerem-se políticas públicas para melhoria na qualidade de vida, saúde, transporte e lazer; além disso, pode-se aventar a possibilidade de parcerias entre comunidade universitária e sociedade local no que tange à elaboração de programas conjuntos que possam promover saúde, prevenir doenças, além de projetos sociais de apoio ao jovem estudante.

No entanto há uma limitação de estudos dessa temática, sendo necessárias outras e mais aprofundadas investigações para ampliar as possibilidades de intervenção no contexto atual.

\section{Agradecimentos}

Ao apoio da Fundação de Amparo à Pesquisa do Estado do Amazonas (FAPEAM), com a concessão da Bolsa de estudo; ao Instituto de Natureza e Cultura- INC-BC da Universidade Federal do Amazonas - UFAM pelo apoio à formação de TAES- Técnico Administrativo em Educação.

\section{Contribuições dos autores}

Guimarães MF participou da organização geral, redação do artigo científico e organização da versão final do artigo. Avoglia HRMC participou da co-orientação do artigo. Vizzotto MM participou da revisão e organização do artigo. Paiva EAF participou na redação do resumo do artigo científico.

\section{Conflitos de interesses}

Nenhum conflito financeiro, legal ou político envolvendo terceiros (governo, empresas e fundações privadas etc.) foi declarado para nenhum aspecto do trabalho submetido (incluindo, mas não se limitando a subvenções e financiamentos, participação em conselho consultivo, desenho de estudo, preparação de manuscrito, análise estatística, etc.).

\section{Referências}

Almeida, M. A. B., Gutierrez, G. L., \& Marques, R. (2012). Qualidade de vida. EACH. http://each.uspnet.usp.br/edicoes-each/ qualidade_vida.pdf 
Andrade, K. O., Souza, J. C., Leite, L. R. C., Figueiró, M. T., \& Cunha, B. V. S. (2011). Qualidade de vida em Estudantes de Psicologia. Psicólogo Informação, 15(15), 129-141. http://pepsic.bvsalud.org/scielo.php?script=sci arttext\&pid=S1415-88092011000100009

Apóstolo, J. L. A., Figueiredo, M. H., Mendes, A. C., \& Rodrigues, M. A. (2011). Depressão, ansiedade e estresse em usuários de cuidados primários de saúde. Revista Latino-Americana de Enfermagem, 19(2), 1-06. https://www.scielo.br/j/rlae/a/ GQqtYNjzjMYVKXVPGQL4mJK/?format=pdf\&lang=pt\#: :text=Identificaram\%2Dse\%20n\%C3\%ADveis\%20graves\%20 ou,est $\%$ C3\%A30\%20forte $\% 20$ e $\% 20$ positivamente $\% 20$ associados

Baldassin, S. (2010). Ansiedade e depressão no estudante de medicina: Revisão de estudos brasileiros. Cadernos ABEM, 6(6), 19-26. http://docplayer.com.br/14923626-Ansiedadee-depressao-no-estudante-de-medicina-revisao-deestudos-brasileiros.html

Barreto, L. M. S., Coutinho, M. P. L., \& Ribeiro, C. G. (2009). Qualidade de vida no contexto migratório: um estudo com imigrantes africanos residentes em João Pessoa-PB, Brasil. Mudanças - Psicologia da Saúde, 17(2), 116-122. https:// www.metodista.br/revistas/revistas-ims/index.php/MUD/ article/view/2127/2089

Calais, S. L., Carrara, K., Brum, M. M., Batista, K., Yamada, J. K., \& Oliveira, J. R. S. (2007). Estresse entre calouros e veteranos de jornalismo. Estudos de Psicologia (Campinas), 24(1), 6977. https://doi.org/10.1590/S0103-166X2007000100008

Diagnostic and Statistical Manual of Mental Disorders (2002). Manual diagnóstico e estatístico de transtornos mentais ( $4^{\mathrm{a}}$ ed). Artmed.

Fleck, M. P. A., Borges, Z. N., Bolognesi, G., \& Rocha, N. S. (2003). Desenvolvimento do WHOQOL, módulo espiritualidade, religiosidade e crenças pessoais. Revista de Saúde Pública, 37(4), 446-455. https://doi.org/10.1590/S0034$\underline{89102003000400009}$

Fleck, M. P. A., Leal, O. F., Louzada, S., Xavier, M., Chachamovich, E., Vieira, G., Santos, L., \& Pinzon, V. (1999). Desenvolvimento da versão em português do instrumento de avaliação de qualidade de vida da OMS (WHOQOL-100). Revista Brasileira de Psiquiatria, 21(1), 19-28. https://doi. org/10.1590/S1516-44461999000100006

Gama, M. M. A., Moura. G. S., Araújo, R. F., \& Teixeira-Silva, F. (2008). Ansiedade-traço em estudantes universitários de Aracaju (SE). Revista de Psiquiatria do Rio Grande do Sul, 30(1),19-24. https://doi.org/10.1590/S0101$\underline{81082008000100007}$
Gardner, J., \& Bell, A. H. (2011). Superando a ansiedade, o pânico e a depressão: Novas maneiras de recuperar a Autoconfiança. Madras Ltda.

Instituto Brasileiro de Geografia e Estatística. (2011). PNAD 2011. https://www.ibge.gov.br/graficos_dinamicos/pnad2011/

Leahy, R. L. (2015). Vença a depressão antes que ela vença você. Artmed.

Lipp, M. N. (2007). Transtorno de Adaptação. Boletim Academia Paulista de Psicologia, 1(7), 72-82. https://www.redalyc.org/ pdf/946/94627112.pdf

Lopes Neto, D., Vieira, H. W. D., Arruda, A. T., \& Farina, H. A. D. (2009). Atenção à Saúde Mental no Amazonas, Brasil: um olhar sobre os Centros de Atenção Psicossocial. Cadernos Brasileiros de Saúde Mental, 1(2), 23-37. https://periodicos. ufsc.br/index.php/cbsm/article/view/68470

Marques, R. F. R. (2007). Esporte e qualidade de vida: reflexão sociológica [tese de mestrado, Universidade Estadual de Campinas]. Red de Repositorios Latinoamericanos. https://repositorioslatinoamericanos.uchile.cl/ handle/2250/1312566

Meyer, C., Guimarães, A. C. A., Machado, Z., \& Parcias, S. R. (2012). Qualidade de vida e estresse ocupacional em estudantes de medicina. Revista Brasileira de Educação Médica, 36(4), 489-498. http://dx.doi.org/10.1590/50100$\underline{55022012000600007}$

Ministério da Sáude. (2010). Departamento de Atenção Básica: Diretrizes do NASF: Núcleo de Apoio a Saúde da Família. http://bvsms.saude.gov.br//publicacoes/diretrizes_do_ nasf_nucleo.pdf

Moreno, D. H., Moreno, R.A., \& Souza, M. G. S. (2012). Transtorno depressivo ao longo da vida. In O. V. Forlenza, \& E. C Miguel (Orgs). Compêndio de Clínica Psiquiátrica. Manole.

Oliveira, J. A. C., \& Oliveira, L. C. (2011). Qualidade de vida: evolução do conceito. In J. C. Souza (Org). Qualidade de vida e Saúde. Vetor.

Organização Mundial da Saúde (1998). WHOQOL e espiritualidade religiosidade e crenças pessoais (SRPB). https://apps.who. int/iris/bitstream/handle/10665/70897/WHO_MSA_ MHP 98.2 eng.pdf; sequence $=1$

Organização Mundial da Saúde (2001). Saúde mental: nova concepção, nova esperança. https://www.who.int/ whr/2001/en/whr01_djmessage_po.pdf

Organização Mundial da Saúde. (2020). Depressão. https://www. paho.org/pt/topicos/depressao 
Parreira, J. (2008). Ansiedade tem cura. Imprimatur.

Petrini, A.C., Margato. G., \& Martins, G.C. (2011). Avaliação da qualidade de vida de estudantes universitários de diferentes períodos: noturno e diurno. $9^{\circ}$ Mostra Acadêmica da UNIMEP. http://www.unimep.br/phpg/mostraacademica/ anais/9mostra/5/348.pdf

Rea, L. M., \& Parker, R.A. (2002). Metodologia de pesquisa: do planejamento à execução. Pioneira Thompson Learning.

Ribeiro, J. L. P., Honrado, A., \& Leal, I. (2004). Contribuição para o estudo da adaptação portuguesa das escalas de ansiedade, depressão e stress (EADS) de 21 itens de Lovibond e Lovibond. Psicologia, Saúde e Doença, 5(2), 229239. https://www.redalyc.org/pdf/362/36250207.pdf

Rossetti Junior, H., \& Schimiguel, J. (2014). Perfil econômico e social dos estudantes de cursos superiores de tecnologia em instituições particulares de ensino. Revista Académica de Economia. http://www.eumed.net/cursecon/ecolat/ br/11/rijs.html
Sociedade Brasileira de Clínica Médica. (2009). Depressão será a doença mais comum do mundo em 2030, diz OMS. https:// www.sbcm.org.br/v2/index.php?catid=0\&id=1317

Souza, A. B., \& Cruz, J. P. (2008). Narrativa: Protótipo da depressão. Mudanças- Psicologia da Saúde, 16(1), 71-80. https://doi.org/10.15603/2176-1019/mud.v16n1p71-80

Souza, L. A., Ines, L. L., Paiva, T. A., Melo, J. G., \& Rechenchosky, L. (2012). Qualidade de vida de acadêmicos de educação física: ingressantes e concluintes. Coleção Pesquisa em Educação Física, 11(5), 129-136. https://fontouraeditora. com.br/periodico/upload/artigo/989 1503352138.pdf

Viana, M. C., \& Andrade, L. H. (2012). Prevalência de transtornos psiquiátricos distribuídos por idade e sexo na Região Metropolitana de São Paulo, Brasil: resultados do Estudo Epidemiológico de Transtornos Mentais São Paulo Megacity. Revista Brasileira de Psiquiatria, 34(3), 249-260. https://doi.org/10.1016/j.rbp.2012.03.001

World Health Organization. (2008). Classificação dos transtornos mentais e de comportamento da CID10. Artmed. 